\title{
The Influence of Shunt Active Power Filter on Stability and Performance in Considering Time-Delay
}

\author{
Tao Li and Yongqiang Liu \\ School of Electric Power, South China University of Technology, Guangzhou, Guangdong 510641, China \\ Correspondence should be addressed to Tao Li; t.l@mail.scut.edu.cn
}

Received 16 August 2012; Revised 15 February 2013; Accepted 4 March 2013

Academic Editor: Yuri Vladimirovich Mikhlin

Copyright ( 2013 T. Li and Y. Liu. This is an open access article distributed under the Creative Commons Attribution License, which permits unrestricted use, distribution, and reproduction in any medium, provided the original work is properly cited.

\begin{abstract}
The existence of time-delay in active power filter (APF) has a great influence on its stability and performance. Hence, this paper deals with the problems of solving the delay margin, the maximal delay which allows the closed-loop system to retain stable, and minimizing the performance index for a given Ho controller. Firstly, for the given time-delay system and controller, a LyapunovKrasovskii functional is used to derive a delay-dependent stability criterion. And a modified Lyapunov-Krasovskii functional is used to obtain less conservative results. Secondly, the mathematical model of APF with time-delay is built, and the two problems already mentioned can be dealt with via transforming them into one of the basic problems of linear matrix inequality (LMI). Finally, the relationships between the delay stability margin and some parameters have been investigated, and the performance index has been discussed in considering time-delay. The effectiveness of the proposed method is verified by simulation studies.
\end{abstract}

\section{Introduction}

In recent years, electrical distribution systems have been suffering from increasing harmonic propagation generated by nonlinear loads. These nonlinear loads draw nonsinusoidal currents from the utility, causing interference with the near sensitive loads and limiting the use of the available electrical supply. The quality of the electrical current thus becomes a significant concern for the distributors of energy and their customers [1].

Different mitigation solutions have been proposed and used, involving passive filters, active power filters (APFs), and hybrid active-passive filters. In general, APFs can be connected in series or in parallel to the nonlinear loads. Shunt active power filters are the most widely used solution. Recently, much work has been done in this area. The key research directions about APF contain topological structure, control strategy, compensation characteristics, resonant depression, harmonic current detection method, and controller design, and so on [2-5]. However, the time-delay in APF is less mentioned, and sometimes it cannot be ignored, even if the time-delay is very small [6]. From the point view of stability analysis, it is important to find the maximal delay time, defined as a delay stability margin, under which an APF system with time-delay can retain stable. In [7], timedelay has a great influence on the stability of APF, but the delay stability margin has not been discussed. The time-delay is an important issue that needs careful treatment to avoid poor performance or even possible instability of the closedloop system [8]. Therefore, the issue of stability of timedelay systems has been widely considered (see, e.g., [9-12]). They can be divided into two classes: delay-dependent and delay-independent. In general, the delay-dependent stability criterion is less conservative than the delay-independent criterion when the size of delay is small.

In this paper, we will deal with the problems of solving the delay margin and minimizing the performance index for a given $\mathrm{H} \infty$ controller. In order to obtain less conservative results, a modified Lyapunov-Krasovskii functional is used and a new criterion is obtained. Then the two problems already mentioned can be dealt with. The relationships between the delay stability margin and some parameters have been investigated, and the performance index has been discussed in considering time-delay.

The notations used in this paper are as follows: $R^{n}$ denotes the real vector space of dimension $n ; R^{m \times n}$ is 
the set of all $m \times n$ real matrices; $A^{T}$ denotes the transpose of matrix $A$. For symmetric matrices $A$ and $B$, the notation $A>B(A \geq B)$ means that matrix $A-B$ is symmetric and positive definite (symmetric and positive semidefinite); $I$ is an identity matrix of appropriate dimensions; The notation $\|\cdot\|_{2}$ stands for the usual $L_{2}[0, \infty)$ norm. For an arbitrary matrix $Z$ and two symmetric matrices $P$ and $Q$, the symmetric term in a symmetric matrix is denoted by $*$; that is,

$$
\left[\begin{array}{ll}
P & Z \\
* & Q
\end{array}\right]=\left[\begin{array}{cc}
P & Z \\
Z^{T} & Q
\end{array}\right]
$$

\section{Two Stability Criteria}

In this section, we will establish two stability criteria. The following lemmas are useful in deriving the criteria.

Lemma 1 (see [13]). For any constant matrix $Z \in R^{n \times n}, Z=$ $Z^{T}>0$, scalar $h>0$, and vector function $x:[-h, 0] \rightarrow R^{n}$, such that the integration in the following is well defined; then

$$
\begin{aligned}
& -h \int_{t-h}^{t} \dot{x}^{T}(s) Z \dot{x}(s) d s \\
& \quad \leq\left[\begin{array}{c}
x(t) \\
x(t-h)
\end{array}\right]^{T}\left[\begin{array}{cc}
-Z & Z \\
Z & -Z
\end{array}\right]\left[\begin{array}{c}
x(t) \\
x(t-h)
\end{array}\right] .
\end{aligned}
$$

Lemma 2 (see [14]). For the symmetric matrix

$$
S=S^{T}=\left[\begin{array}{cc}
S_{11} & S_{12} \\
* & S_{22}
\end{array}\right], \quad S \in R^{n \times n}, S_{11} \in R^{r \times r}(r<n),
$$

the three conditions are equivalent to each other:

(1) $S<0$;

(2) $S_{11}<0, S_{22}-S_{12}^{T} S_{11}^{-1} S_{12}<0$;

(3) $S_{22}<0, S_{11}-S_{12} S_{22}^{-1} S_{12}^{T}<0$.

Consider the following time-invariant system with the input delay and disturbance:

$$
\begin{gathered}
\dot{x}(t)=A x(t)+B_{1} u(t-\tau)+w(t), \\
z(t)=C x(t), \\
x(t)=\phi(t), \quad \forall t \in[-\tau, 0], \\
\int_{0}^{\infty} w^{T}(t) w(t) d t<\infty,
\end{gathered}
$$

that is,

$$
w(t) \in L_{2}[0, \infty)
$$

where $x(t) \in R^{n}$ is the state vector, $w(t) \in R^{n}$ is the disturbance vector, $u(t) \in R^{n}$ is the control input, $z(t) \in R^{n}$ is the controlled output, and $A, B_{1}$, and $C$ are constant matrices of appropriate dimensions, $\tau>0$ is the constant delay, and $\phi(t)$ is the initial condition.
For a given Hoo controller,

$$
u(t)=K x(t)
$$

where $K$ is the state feedback gain matrix to be given.

For the controller (7), it satisfies the following two conditions:

(1) the closed-loop system is asymptotically stable;

(2) the closed-loop system guarantees, under zero initial condition, $\|z(t)\|_{2}<\gamma\|w(t)\|_{2}$ for all nonzero $w(t) \epsilon$ $L_{2}[0, \infty)$ and some performance index constant $\gamma>$ 0 .

Then the system (4) with the given controller is rewritten as

$$
\begin{gathered}
\dot{x}(t)=A x(t)+B x(t-\tau)+w(t), \\
z(t)=C x(t), \\
x(t)=\phi(t), \quad \forall t \in[-\tau, 0],
\end{gathered}
$$

where $B=B_{1} K$.

We have the following result.

Proposition 3. For a given scalar $\tau>0$, the closed-loop system described by (8) is asymptotically stable with Ho performance index $\gamma>0$ for any given $\tau$, if there exist real symmetrical $n \times n$ matrices $P=P^{T}>0, Q=Q^{T}>0$, and $Z=Z^{T}>0$ such that $\Omega=\Omega^{T}<0$, where $A, B$, and $C$ are constant matrices, $\lambda=\gamma^{2}$, and

$\Omega=\left(\begin{array}{cccc}A^{T} P+P A+Q-Z+C^{T} C & P B+Z & P & \tau A^{T} Z \\ * & -Q-Z & 0 & \tau B^{T} Z \\ * & * & -\lambda I & \tau Z \\ * & * & * & -Z\end{array}\right)$.

Proof. Choose a Lyapunov-Krasovskii functional candidate as [15]

$$
\begin{aligned}
V\left(t, x_{t}\right)= & x^{T}(t) P x(t) \\
& +\int_{t-\tau}^{t} x^{T}(s) Q x(s) d s \\
& +\int_{-\tau}^{0} \int_{t+\theta}^{t} \dot{x}^{T}(s) \tau Z \dot{x}(s) d s d \theta
\end{aligned}
$$

where $x_{t}$ is defined as $x_{t}=x(t+\theta)$, for all $\theta \in[-\tau, 0]$, and $P=P^{T}>0, Q=Q^{T}>0$, and $Z=Z^{T}>0$ are matrices 
to be chosen. Taking the derivative of $V\left(t, x_{t}\right)$ with respect to talong the trajectory of (8) yields

$$
\begin{aligned}
\dot{V}\left(t, x_{t}\right)= & {\left[\dot{x}^{T}(t) P x(t)+x^{T}(t) P \dot{x}(t)\right] } \\
& +\left[x^{T}(t) Q x(t)-x^{T}(t-\tau) Q x(t-\tau)\right] \\
& +\left[\tau^{2} \dot{x}^{T}(t) Z \dot{x}(t)-\tau \int_{t-\tau}^{t} \dot{x}^{T}(s) Z \dot{x}(s) d s\right] \\
= & x^{T}(t)\left(A^{T} P+P A+Q\right) x(t) \\
& +x^{T}(t) P B x(t-\tau)+x^{T}(t) P w(t) \\
& +x^{T}(t-\tau) B^{T} P x(t)-x^{T}(t-\tau) Q x(t-\tau) \\
& +w^{T}(t) P x(t)+\tau^{2} \dot{x}^{T}(t) Z \dot{x}(t) \\
& -\tau \int_{t-\tau}^{t} \dot{x}^{T}(s) Z \dot{x}(s) d s .
\end{aligned}
$$

Use Lemma 1 to obtain

$$
\begin{aligned}
& -\tau \int_{t-\tau}^{t} \dot{x}^{T}(s) Z \dot{x}(s) d s \\
& \leq\left[\begin{array}{c}
x(t) \\
x(t-\tau)
\end{array}\right]^{T}\left[\begin{array}{cc}
-Z & Z \\
Z & -Z
\end{array}\right]\left[\begin{array}{c}
x(t) \\
x(t-\tau)
\end{array}\right] \\
& =-x^{T}(t) Z x(t)+x^{T}(t) Z x(t-\tau) \\
& \quad+x^{T}(t-\tau) Z x(t)-x^{T}(t-\tau) Z x(t-\tau) .
\end{aligned}
$$

Then, the following holds:

$$
\begin{aligned}
\dot{V}\left(t, x_{t}\right) \leq & x^{T}(t)\left(A^{T} P+P A+Q-Z+\tau^{2} A^{T} Z A\right) x(t) \\
& +x^{T}(t)\left(P B+Z+\tau^{2} A^{T} Z B\right) x(t-\tau) \\
& +x^{T}(t)\left(P+\tau^{2} A^{T} Z\right) w(t) \\
& +x^{T}(t-\tau)\left(B^{T} P+Z+\tau^{2} B^{T} Z A\right) x(t) \\
& +x^{T}(t-\tau)\left(-Q-Z+\tau^{2} B^{T} Z B\right) x(t-\tau) \\
& +\tau^{2} x^{T}(t-\tau) B^{T} Z w(t) \\
& +w^{T}(t)\left(P+\tau^{2} Z A\right) x(t)+\tau^{2} w^{T}(t) Z B x(t-\tau) \\
& +\tau^{2} w^{T}(t) Z w(t) .
\end{aligned}
$$

Assuming zero initial condition, we have $\left.V\left(t, x_{t}\right)\right|_{t=0}=0$.

Consider the following index:

$$
J_{z w} \triangleq \int_{0}^{\infty}\left[z^{T}(t) z(t)-\gamma^{2} w^{T}(t) w(t)\right] d t .
$$

Then, for any nonzero $w(t) \in L_{2}[0, \infty)$ there holds

$$
\begin{aligned}
J_{z w} \leq & \int_{0}^{\infty}\left[z^{T}(t) z(t)-\gamma^{2} w^{T}(t) w(t)\right] d t \\
& +\left.V\left(t, x_{t}\right)\right|_{t=\infty}-\left.V\left(t, x_{t}\right)\right|_{t=0} \\
= & \int_{0}^{\infty}\left[z^{T}(t) z(t)-\gamma^{2} w^{T}(t) w(t)+V\left(t, x_{t}\right)\right] d t \\
\leq & \int_{0}^{\infty} \Gamma^{T} \Omega_{1} \Gamma d t,
\end{aligned}
$$

where $\Gamma^{T}=\left(x^{T}(t), x^{T}(t-\tau), w^{T}(t)\right)$ and

$$
\begin{gathered}
\Omega_{1}=\left[\begin{array}{ccc}
\Psi_{11} & P B+Z+\tau^{2} A^{T} Z B & P+\tau^{2} A^{T} Z \\
* & -Q-Z+\tau^{2} B^{T} Z B & \tau^{2} B^{T} Z \\
* & * & -\gamma^{2} I+\tau^{2} Z
\end{array}\right], \\
\Psi_{11}=A^{T} P+P A+Q-Z+C^{T} C+\tau^{2} A^{T} Z A .
\end{gathered}
$$

When only considering the stability of the closed-loop system (8), we can assume the disturbance input is zero; that is, $w(t) \equiv 0$, and if the matrix $\Omega_{1}$ is negative definite, we can obtain $\dot{V}\left(t, x_{t}\right)<0$ and the asymptotic stability of system (8) is established. When $w(t) \in L_{2}[0, \infty)$ and $\Omega_{1}<0$, then we can obtain $J_{z w}<0$, and therefore $\|z(t)\|_{2}<\gamma\|w(t)\|_{2}$.

We know

$$
\begin{aligned}
& \Omega_{1}=\left[\begin{array}{ccc}
\Psi_{11} & P B+Z+\tau^{2} A^{T} Z B & P+\tau^{2} A^{T} Z \\
* & -Q-Z+\tau^{2} B^{T} Z B & \tau^{2} B^{T} Z \\
* & * & -\gamma^{2} I+\tau^{2} Z
\end{array}\right] \\
& =\left[\begin{array}{ccc}
\Psi_{1} & P B+Z & P \\
* & -Q-Z & 0 \\
* & * & -\gamma^{2} I
\end{array}\right]+\left[\begin{array}{c}
A^{T} \\
B^{T} \\
I
\end{array}\right] \tau^{2} Z\left[\begin{array}{lll}
A & B & I
\end{array}\right] \\
& =\left[\begin{array}{ccc}
\Psi_{1} & P B+Z & P \\
* & -Q-Z & 0 \\
* & * & -\gamma^{2} I
\end{array}\right] \\
& +\left[\begin{array}{c}
\tau A^{T} Z \\
\tau B^{T} Z \\
\tau Z
\end{array}\right](-Z)^{-1}\left[\begin{array}{llll}
\tau A & \tau Z B & \tau Z
\end{array}\right] \\
& =\left[\begin{array}{ccc}
\Psi_{1} & P B+Z & P \\
* & -Q-Z & 0 \\
* & * & -\gamma^{2} I
\end{array}\right]-\Psi_{12}(-Z)^{-1} \Psi_{12}^{T} \text {, }
\end{aligned}
$$

where $Z=Z^{T}>0, \Psi_{1}=A^{T} P+P A+Q-Z+C^{T} C, \Psi_{12}^{T}=$ $\left[\begin{array}{lll}\tau Z A & \tau Z B & \tau Z\end{array}\right]$

$$
\begin{gathered}
\Psi_{1}=A^{T} P+P A+Q-Z+C^{T} C, \\
\Psi_{12}^{T}=\left[\begin{array}{lll}
\tau Z A & \tau Z B & \tau Z
\end{array}\right] .
\end{gathered}
$$

From the Schur complement (Lemma 2), $\Omega_{1}<0$ is equivalent to $\Omega<0$. This completes the proof.

Proposition 4. For a given scalar $\tau>0$, the closed-loop system described by (8) is asymptotically stable with Ho performance 
index $\gamma>0$ for any given $\tau$, if there exist real symmetrical $n \times n$ matrices $P=P^{T}>0, Q=Q^{T}>0, Z=Z^{T}>0, W=W^{T} \geq 0$, $S_{11}=S_{11}^{T}$, and $S_{22}=S_{22}^{T}$ such that

$$
\begin{gathered}
S=\left(\begin{array}{ll}
S_{11} & S_{12} \\
S_{12}^{T} & S_{22}
\end{array}\right) \geq 0, \\
\Phi=\Phi^{T}<0,
\end{gathered}
$$

where

$$
\begin{gathered}
\Phi=\left(\begin{array}{cccccc}
\Phi_{11} & \Phi_{12} & P B+Z & P & \frac{\tau}{2} A^{T} W & \tau A^{T} Z \\
* & \Phi_{22} & -S_{12} & 0 & 0 & 0 \\
* & * & \Phi_{33} & 0 & \frac{\tau}{2} B^{T} W & \tau B^{T} Z \\
* & * & * & -\lambda I & \frac{\tau}{2} W & \tau Z \\
* & * & * & * & -W & 0 \\
* & * & * & * & * & -Z
\end{array}\right), \\
\Phi_{11}=A^{T} P+P A+Q+S_{11}-W-Z+C^{T} C, \\
\Phi_{12}=W+S_{12}, \\
\Phi_{33}=-S_{22}-Q-Z,
\end{gathered}
$$

Proof. Choose a new Lyapunov-Krasovskii functional candidate as follows [16]:

$$
\begin{aligned}
V\left(t, x_{t}\right)= & x^{T}(t) P x(t)+\int_{t-\tau}^{t} x^{T}(s) Q x(s) d s \\
& +\int_{-\tau}^{0} \int_{t+\theta}^{t} \dot{x}^{T}(s) \tau Z \dot{x}(s) d s d \theta \\
& +\int_{t-(\tau / 2)}^{t} y^{T}(s) S y(s) d s \\
& +\int_{-\tau / 2}^{0} \int_{t+\theta}^{t} \dot{x}^{T}(s) \frac{\tau}{2} W \dot{x}(s) d s d \theta
\end{aligned}
$$

where $x_{t}$ is defined as $x_{t}=x(t+\theta)$, for all $\theta \in[-\tau, 0]$, and

$$
\begin{gathered}
P=P^{T}>0, \quad Q=Q^{T}>0, \quad Z=Z^{T}>0, \\
W=W^{T} \geq 0, \\
S=S^{T}=\left(\begin{array}{ll}
S_{11} & S_{12} \\
S_{12}^{T} & S_{22}
\end{array}\right) \geq 0, \\
y^{T}(s)=\left(\begin{array}{ll}
x^{T}(s) & \left.x^{T}\left(s-\frac{\tau}{2}\right)\right) .
\end{array}\right.
\end{gathered}
$$

The later proof is omitted due to the fact that it is much similar to the proof of Proposition 3.

Remark 5. If we only consider the stability of the closedloop system (8), we can ignore the performance index $\gamma$. In

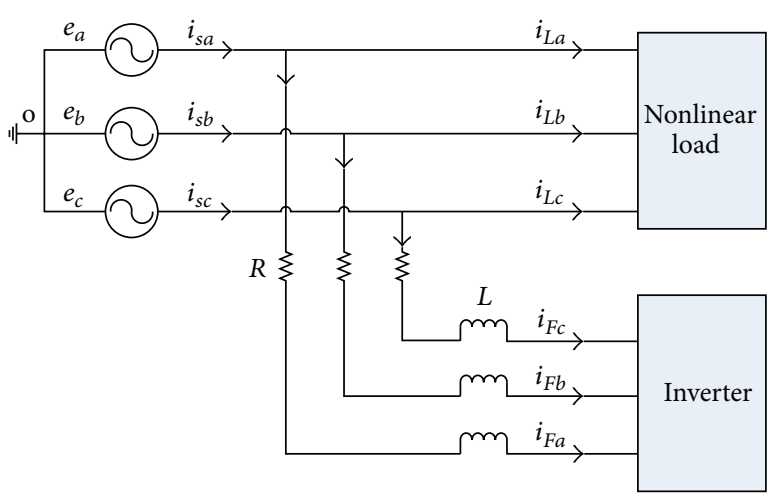

FIGURE 1: Circuit configuration of shunt active power filter.

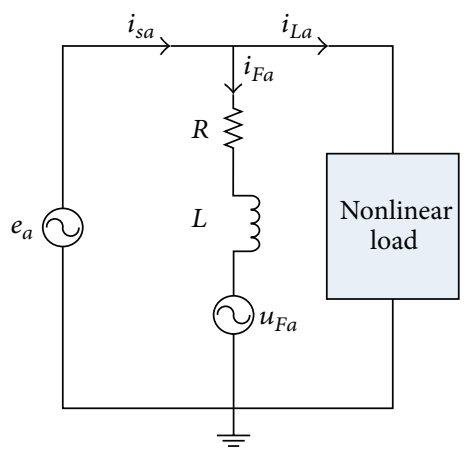

FIgURE 2: Equivalent circuit topology of the A phase.

other words, the performance index $\gamma$ can be considered as a variable, not a given constant in the two propositions.

Remark 6. The choice of the Lyapunov-Krasovskii functional is very important to the conservation of the derived results. When we uniformly divide the delay interval $[-\tau, 0]$ into two subintervals $[-\tau,-\tau / 2]$ and $[-\tau / 2,0]$, we obtain the new functional in Proposition 4. Similarly, if we uniformly divide the delay interval $[-\tau, 0]$ into $N$ subintervals and $N$ is a positive integer, the derived result has less conservation for a bigger $N$ by using the obtained functional. However, as $N$ increases, testing the result is much time consuming. One can employ the result in the case of $N=2$ (Proposition 4) for the tradeoff between better results and time consuming ones [16].

\section{Mathematical Model of APF with Time-Delay}

The circuit configuration of shunt active power filter consisting of a filter inductor in series with PWM inverter is shown in Figure 1. Assume the three-phase source voltage is symmetrical and the equivalent circuit topology of the single phase is shown in Figure 2. Source voltage, source current, load current, filter side current, and the output voltage of inverter are represented by $e_{a}, i_{S a}, i_{L a}, i_{F a}$, and $u_{F a}$, respectively. 
Basic equation of this circuit is derived by Kirchhoff's voltage law:

$$
\dot{L i_{F a}}=e_{a}-R i_{F a}-u_{F a} .
$$

And the equations of the other two phases are similar.

When the control delay is considered and $\tau$ stands for the total delay, then

$$
\dot{L i}_{F a b c}(t)=e_{a b c}(t)-R i_{F a b c}(t)-u_{F a b c}(t-\tau),
$$

where

$$
\begin{gathered}
i_{F a b c}=\left(i_{F a}, i_{F b}, i_{F c}\right)^{T}, \\
e_{a b c}=\left(e_{a}, e_{b}, e_{c}\right)^{T}, \\
u_{F a b c}=\left(u_{F a}, u_{F b}, u_{F c}\right)^{T} .
\end{gathered}
$$

Using Park's transformation to (19), the three-phase quantities are transformed into a synchronous rotating reference frame. The general transformation matrix is

$$
\begin{aligned}
C_{a b c \rightarrow d q}(t) & =\sqrt{\frac{2}{3}}\left[\begin{array}{ccc}
\cos \omega t & \cos \left(\omega t-\frac{2}{3} \pi\right) & \cos \left(\omega t+\frac{2}{3} \pi\right) \\
\sin \omega t & \sin \left(\omega t-\frac{2}{3} \pi\right) & \sin \left(\omega t+\frac{2}{3} \pi\right)
\end{array}\right]
\end{aligned}
$$

and $C_{d q \rightarrow a b c}(t)=C_{a b c \rightarrow d q}^{T}(t)$.

Then we obtain the transformed model in the synchronous orthogonal rotating frame as follows:

$$
\left[\begin{array}{c}
\dot{i}_{F d} \\
\dot{i}_{F q}
\end{array}\right]=A\left[\begin{array}{l}
i_{F d} \\
i_{F q}
\end{array}\right]+B(\tau)\left[\begin{array}{l}
u_{F d}(t-\tau) \\
u_{F q}(t-\tau)
\end{array}\right]+\left[\begin{array}{l}
e_{d} \\
e_{q}
\end{array}\right],
$$

where

$$
\begin{gathered}
A=\left[\begin{array}{rr}
-\frac{R}{L} & -\omega \\
\omega & -\frac{R}{L}
\end{array}\right], \\
B(\tau)=-\frac{1}{L}\left[\begin{array}{cc}
\cos \omega \tau & -\sin \omega \tau \\
\sin \omega \tau & \cos \omega \tau
\end{array}\right] .
\end{gathered}
$$

Note that $i_{F d}=i_{F f d}+i_{F h d}, i_{F q}=i_{F f q}+i_{F h q}$; then

$$
\begin{aligned}
{\left[\begin{array}{c}
i_{F f d}+i_{F h d} \\
i_{F f q}+i_{F h q}
\end{array}\right]=} & A\left[\begin{array}{l}
i_{F f d}+i_{F h d} \\
i_{F f q}+i_{F h q}
\end{array}\right] \\
& +B(\tau)\left[\begin{array}{l}
u_{F f d}(t-\tau)+u_{F h d}(t-\tau) \\
u_{F f q}(t-\tau)+u_{F h q}(t-\tau)
\end{array}\right]+\left[\begin{array}{l}
e_{d} \\
e_{q}
\end{array}\right],
\end{aligned}
$$

where the fundamental frequency component of filter current in the synchronous orthogonal rotating frame and the relevant harmonic component are represented by $i_{F f d}, i_{F f q}, i_{F h d}$, and $i_{F h q}$ respectively. The other variables are similar.
$[4]$

Here the output voltage function of inverter is chosen as

$$
u_{F}=K i_{S h}+e,
$$

where $K>0$.

The system (30) is a linear system, so it satisfies superposition principle: The last equation can be rewritten as follows.

(1) Only consider the fundamental frequency component,

$$
\begin{gathered}
u_{F f}=e, \\
{\left[\begin{array}{ll}
u_{F f d} & u_{F f q}
\end{array}\right]=\left[\begin{array}{ll}
e_{d} & e_{q}
\end{array}\right]=\left[\begin{array}{ll}
E & 0
\end{array}\right],} \\
{\left[\begin{array}{l}
i_{F f d} \\
i_{F f q}
\end{array}\right]=A\left[\begin{array}{l}
i_{F f d} \\
i_{F f q}
\end{array}\right]+B(\tau)\left[\begin{array}{l}
u_{f d}(t-\tau) \\
u_{f q}(t-\tau)
\end{array}\right]+\frac{1}{L}\left[\begin{array}{l}
e_{d} \\
e_{q}
\end{array}\right] .}
\end{gathered}
$$

The last equation above can be rewritten as

$$
0=A\left[\begin{array}{c}
i_{F f d} \\
i_{F f q}
\end{array}\right]+B(\tau)\left[\begin{array}{c}
E \\
0
\end{array}\right]+\frac{1}{L}\left[\begin{array}{c}
E \\
0
\end{array}\right] .
$$

This implies

$$
\left[\begin{array}{l}
i_{F f d} \\
i_{F f q}
\end{array}\right]=\frac{E L}{R^{2}+\omega^{2} L^{2}}\left[\begin{array}{cc}
\frac{R}{L} & -\omega \\
\omega & \frac{R}{L}
\end{array}\right]\left[\begin{array}{c}
1-\cos \omega \tau \\
-\sin \omega \tau
\end{array}\right] .
$$

In other words, the fundamental frequency component of filter current exists because of the time-delay.

(2) Only consider the harmonic frequency component.

$$
\left[\begin{array}{l}
\dot{i}_{\text {Fhd }} \\
\dot{i}_{\text {Fhq }}
\end{array}\right]=A\left[\begin{array}{l}
i_{\text {Fhd }} \\
i_{\text {Fhq }}
\end{array}\right]+B(\tau)\left[\begin{array}{l}
u_{h d}(t-\tau) \\
u_{h q}(t-\tau)
\end{array}\right] .
$$

The equilibrium point is as follows:

$$
\left[\begin{array}{ll}
i_{\text {Fhd }}^{*} & i_{\text {Fhq }}^{*}
\end{array}\right]^{T}=\left[\begin{array}{ll}
-i_{\text {Lhd }} & -i_{\text {Lhq }}
\end{array}\right]^{T} .
$$

The error model is obtained:

$$
\left[\begin{array}{l}
i_{\text {Shd }}^{\cdot} \\
i_{\text {Shq }}
\end{array}\right]=A\left[\begin{array}{l}
i_{\text {Shd }} \\
i_{\text {Shq }}
\end{array}\right]+B(\tau)\left[\begin{array}{l}
u_{h d}(t-\tau) \\
u_{h q}(t-\tau)
\end{array}\right]+w(t),
$$

where

$$
\begin{gathered}
w(t)=\left[\begin{array}{l}
\dot{i}_{\text {Lhd }} \\
i_{\text {Lhd }}
\end{array}\right]-A\left[\begin{array}{l}
i_{\text {Lhd }} \\
i_{\text {Lhq }}
\end{array}\right], \\
u_{F h}=K i_{S h} .
\end{gathered}
$$

Then (37) is rewritten as

$$
\begin{gathered}
\dot{x}(t)=A x(t)+B(\tau) K x(t-\tau)+w(t), \\
z(t)=C x(t),
\end{gathered}
$$

where the state variable $x(t)=\left(i_{\text {Shd }} \quad i_{S h q}\right)^{T}$ and $z(t)$ is the controlled output. Matrix $A, B(\tau)$, and $w(t)$ are defined in (29) and (38), $C=\operatorname{diag}\left(\left(K_{0}+R\right) / L,\left(K_{0}+R\right) / L\right)$ because of the big disturbance $w(t)$, and $K_{0}$ is a given constant.

Then the two problems above can be dealt with by converting them into the following optimization problems. 
Problem 1. If we only consider the stability of the APF system, delay stability margin can be solved as follows:

maximize $\tau$

subject to $\Omega<0 \quad(\Phi<0$ for Proposition 4$)$,

$$
\begin{aligned}
& P=P^{T}>0, \quad Q=Q^{T}>0, \\
& Z=Z^{T}>0, \quad \lambda=\gamma^{2}>0, \\
& \left(S=S^{T} \geq 0, W=W^{T} \geq 0 \text { for Proposition } 4\right) .
\end{aligned}
$$

Problem 2. For the given controller and time-delay $\tau$, the relationship between the time-delay and the controller performance can be described by the performance index $\gamma>0$ as follows:

minimize $\lambda=\gamma^{2}$

subject to $\Omega<0 \quad(\Phi<0$ for Proposition 4$)$,

$$
\begin{aligned}
& P=P^{T}>0, \quad Q=Q^{T}>0, \\
& Z=Z^{T}>0, \quad \lambda=\gamma^{2}>0 \\
& \left(S=S^{T} \geq 0, W=W^{T} \geq 0 \text { for Proposition } 4\right) .
\end{aligned}
$$

These convex optimization problems can be transformed into one of the basic problems of linear matrix inequality (LMI) and solved by using feasp function.

\section{Simulation Analysis}

In this section, the two problems already mentioned will be discussed separately, and the MATLAB software is used as the simulation and calculation software. We assume that the three-phase source voltages are symmetrical. A typical threephase rectifier is adopted as the nonlinear loads. In the filter side, $R=0.2 \Omega, L=7.5 \mathrm{mH}, K=100$, and $f=50 \mathrm{HZ}$; then $w=2 \pi f$ and $K_{0}=100$.

Figure 3 shows the simulation results of source current and its harmonic component when $K=100$, and Figure 4 shows the simulation results when $K=50$, under the circumstance of not taking the delay into account. From the comparison of Figures 3 and 4, we know that if the time-delay is omitted, the value of $K$ is bigger, the harmonic current is smaller, and the filtering action is better.

4.1. The Analysis of Delay Stability Margin. Firstly, we only consider the stability by using the method in Problem 1 and then analysing the relationship between delay stability margin and some parameters.

4.1.1. The Algorithm of Solving Delay Stability Margin. For any $\tau$ we can compute the result whether the subject conditions are satisfied, the maximum $\tau$ is what we need.

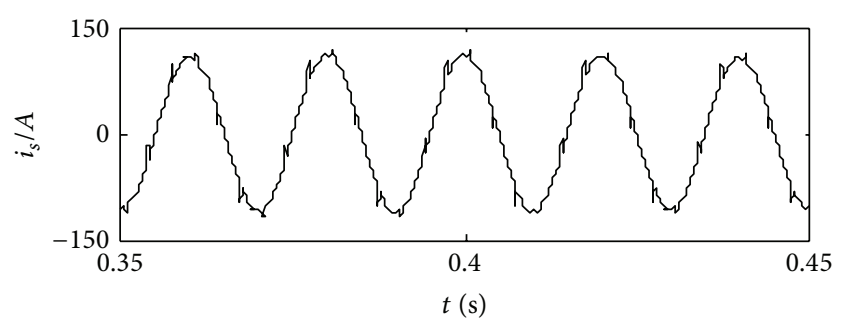

(a)

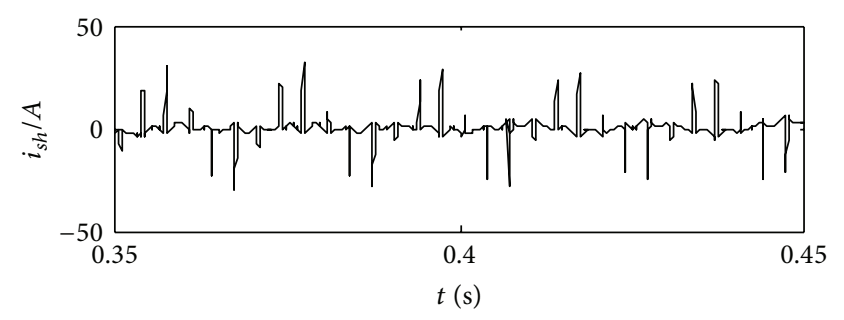

(b)

Figure 3: $K=100$.

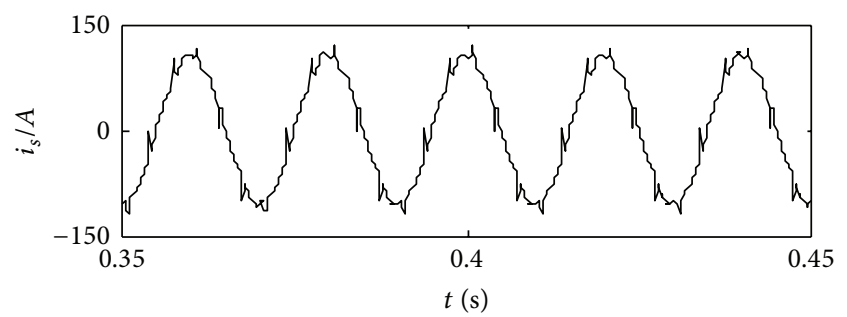

(a)

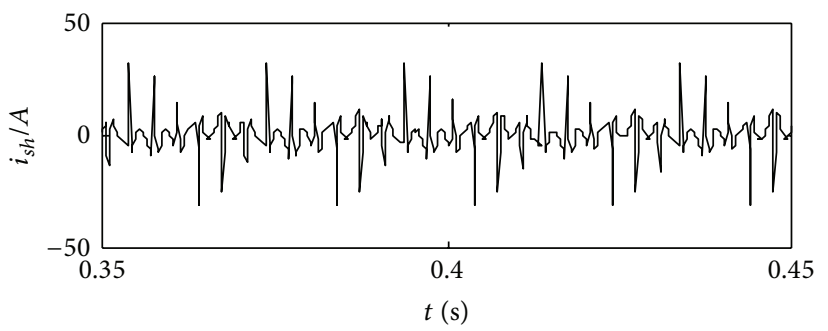

(b)

FIGURE $4: K=50$.

The basic steps are described as follows:

(1) choose a computed precision $\varepsilon$ and assign it to $\tau$ as its initial value;

(2) check whether the LMIs are true. If true, jump to step (3); else stop;

(3) if $\tau_{a}=\tau, \tau_{b}=2 \tau$, then $\tau$ gets its new value of $\tau_{b}$. Check the LMIs again until the result is false;

(4) assign the value of $\left(\tau_{a}+\tau_{b}\right) / 2$ to $\tau$ and check the LMIs. if it is true, $\tau_{a}=\tau$; else $\tau_{b}=\tau$ until $\left(\tau_{b}-\tau_{a}\right) / 2 \leq \varepsilon$;

(5) here if the value of $\tau_{a}$ is the delay stability margin, then stop. 
TABLE 1: The computed value of the delay stability $(K=100, L=$ $7.5 \mathrm{mH})$.

\begin{tabular}{lc}
\hline & $\tau_{\max } / \mu \mathrm{s}$ \\
\hline Proposition 3 & 105.43 \\
Proposition 4 & 114.73 \\
\hline
\end{tabular}

TABLE 2: The calculated value and fitted value for different values of $K,(L=7.5 \mathrm{mH})$

\begin{tabular}{lccccc}
\hline$\tau_{\max } / \mu$ s & $K=20$ & $K=60$ & $K=100$ & $K=140$ & $K=180$ \\
\hline Calculated value & 571.46 & 191.12 & 114.73 & 81.961 & 63.751 \\
Fitted value & 574.00 & 191.33 & 114.80 & 82.000 & 63.778 \\
\hline
\end{tabular}

Taking the above example, for example, and $\varepsilon=10^{(-8)}$, the delay stability margin is obtained by employing Propositions 3 and 4 . The results are listed in Table 1.

Results in Table 1 show that the delay stability margin calculated by Proposition 4 is bigger $(114.73 / 105.43 \approx 1.0882)$. In other word, the derived result by Proposition 4 is less conservative.

Then, the relationships between the delay stability margin and some other parameters will be investigated.

\subsubsection{Delay Stability Margin with Parameter Variation.} Firstly, $L=7.5 \mathrm{mH}$ is fixed, the value of $K$ is increasing from 10 to 200 steadily, and the interval is 10 . The results are shown in Figure 5 and Table 2 by using Proposition 4. When the other parameters are invariant and the value of $K$ is bigger, the filter action is better. However, the delay stability margin is smaller and is in approximate inverse proportion with $K$, so we can employ the fitted curve with an inverse proportion functional. The result is

$$
\tau_{\max }=\frac{0.01148}{K}, \quad(\text { if } L=7.5 \mathrm{mH}) .
$$

The fitted values of some points are listed in Table 2. A good fit is obtained, with the mean deviation between the calculated and fitted values being less than $1 \%$.

Secondly, $K=100$ is fixed, the value of $L$ is increasing from $0.75 \mathrm{mH}$ to $15 \mathrm{mH}$ steadily, and the interval is $0.75 \mathrm{mH}$. Figure 6 shows the results. When the value of filter inductance is increasing, the delay stability margin is changing in approximate proportion, so there is a proportion functional to fit the calculated values. The result is

$$
\tau_{\max }=0.01529 * L, \quad(\text { if } K=100) .
$$

The fitted values of some points are listed in Table 3. A good linear fit is also obtained, with the mean deviation between the calculated and fitted values being less than $1 \%$.

Finally, the value of $K$ is increasing from 10 to 200 steadily and the interval is 10; at the same time, the value of $L$ is increasing from $0.75 \mathrm{mH}$ to $15 \mathrm{mH}$ steadily and the interval is $0.75 \mathrm{mH}$. The number of the group $(K, L)$ is 400 . Noting (42) and (43), the estimated curve between the delay stability and the group $(K, L)$ is obtained as follows:

$$
\tau_{\max }=\alpha * \frac{L}{K},
$$

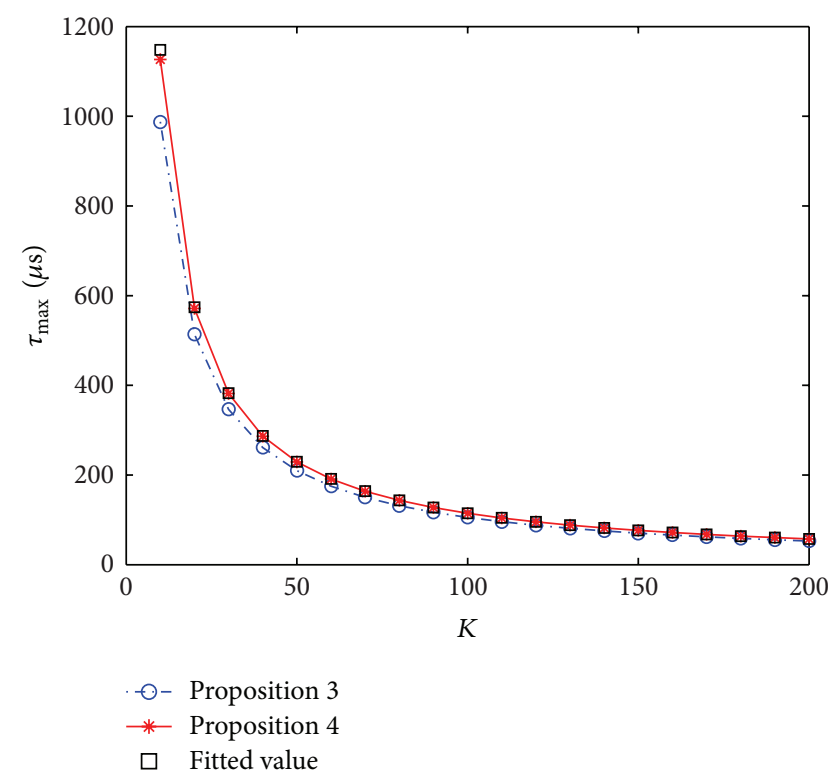

FIgURE 5: The relationship between the delay stability and $K(L=$ $7.5 \mathrm{mH})$.

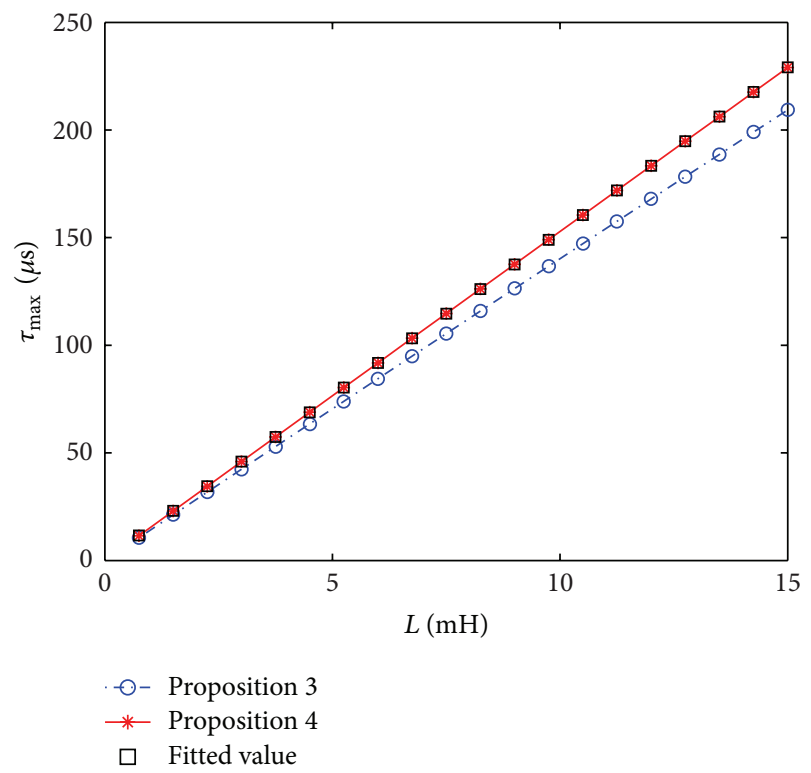

FIgURE 6: The relationship between the delay stability and $L(K=$ 100).

where the value of $\alpha$ is 1.5298 for Proposition 4 and 1.4057 for Proposition 3.

Taking the calculated and estimated values by using Proposition 4; for example, there are ten typical groups listed in Table 4 , where $(a, b)$ stands for the group $(a * K 0, b * L 0)$, and $K 0=10, L 0=0.75 \mathrm{mH}$. The estimated values are very close to the calculated values, and the mean deviation of them is less than $1 \%$.

4.2. The Relationship between the Time-Delay and the Controller Performance. The relationship can be described by 
TABLE 3: The calculated value and fitted value for different values of $L,(K=100, L 0=0.75 \mathrm{mH})$.

\begin{tabular}{lccccc}
\hline$\tau_{\max } / \mu s$ & $L=2 * L 0$ & $6 * L 0$ & $10 * L 0$ & $14 * L 0$ & $18 * L 0$ \\
\hline Calculated value & 22.984 & 68.893 & 114.73 & 160.49 & 206.19 \\
Fitted value & 22.935 & 68.805 & 114.68 & 160.55 & 206.42 \\
\hline
\end{tabular}

TABLE 4: The calculated value and estimated value for different groups $(K, L)$.

\begin{tabular}{lccccc}
\hline$\tau_{\max } / \mu$ s & $(6,2)$ & $(6,6)$ & $(6,10)$ & $(6,14)$ & $(10,6)$ \\
\hline Calculated value & 38.320 & 114.82 & 191.12 & 267.22 & 68.893 \\
Estimated value & 38.245 & 114.735 & 191.225 & 267.715 & 68.841 \\
Relative error & $0.20 \%$ & $0.074 \%$ & $0.055 \%$ & $0.19 \%$ & $0.075 \%$ \\
\hline$\tau_{\max } / \mu$ s & $(10,14)$ & $(14,6)$ & $(14,10)$ & $(14,14)$ & $(14,18)$ \\
\hline Calculated value & 160.490 & 49.207 & 81.961 & 114.68 & 147.38 \\
Estimated value & 160.629 & 49.172 & 81.9536 & 114.82 & 147.516 \\
Relative error & $0.087 \%$ & $0.071 \%$ & $0.01 \%$ & $0.12 \%$ & $0.093 \%$ \\
\hline
\end{tabular}

the performance index $\gamma$, and the derived method has been showen in Problem 2. Similar to the procedure of the delay stability margin, we define $\eta=1 / \lambda=1 / \gamma^{2}$ then we can obtain the minimum performance index $\gamma$ by maximizing the scalar $\eta$ in the given time-delay and controller.

We consider the three kinds of case: without time-delay, a smaller time-delay and a bigger time-delay, that is, $\tau=0 \mu \mathrm{s}$, $\tau=20 \mu \mathrm{s}$, and $\tau=80 \mu \mathrm{s}$. The results are shown in Figure 7 by using Proposition 4 because of the less conservatism, and $K$ is increasing from 10 to 140 steadily and the interval is 10 for each given time-delay. Similarly, we choose three different gains $K$ to describe the relationship between the time-delay and the performance in Figure 8.

It can be seen from Figure 7 that when there is no timedelay on input; that is, $\tau=0 \mu \mathrm{s}$, the performance is better as the increase of $K$. The result is similar to the smaller timedelay. However, when the given time-delay is near to delay stability margin, in other words, the time-delay is bigger, the performance is worse than the middle value of $K$, so a bigger gain is not necessary for a serious time-delay. From Figure 8 we can obtain the similar results. Besides, it is observed clearly that the performance is worse as the increase of the time-delay for all gains and the influence for a small gain is less than the one for a big gain.

\section{Conclusion}

This paper has investigated the delay-dependent stability of $\mathrm{APF}$ and the influence on the performance in considering time-delay. After preliminary analysis of the relationships between the delay stability margin and some other parameters, the result can be estimated by (44) and the estimated values show good agreement with the calculated values. How to obtain a bigger value of $\alpha$ in (44) will be further considered. The delay stability margin can be used as a design objective to guide the selection of different parameters. The time-delay is common in practical applications, and it cannot be predicted 100 percent true, so it is necessary to research the influence.

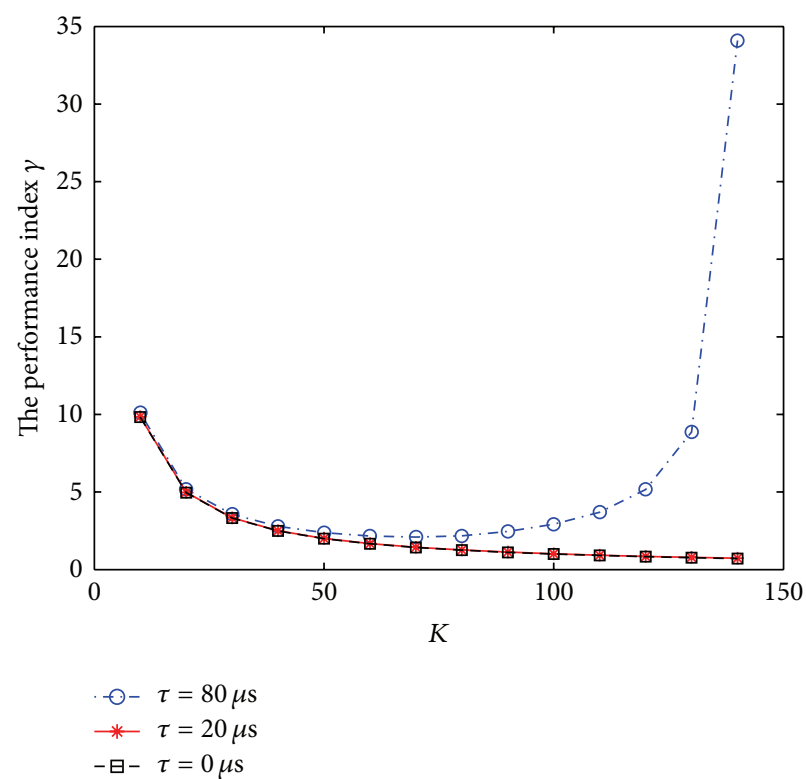

FIGURE 7: The relationship between the gain $K$ and the performance.

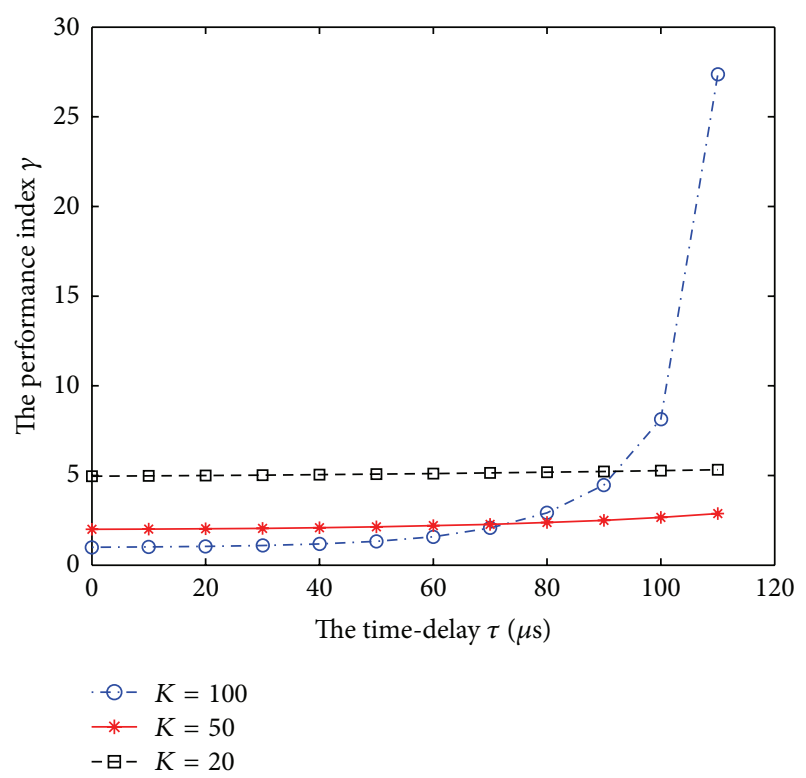

FIGURE 8: The relationship between the time-delay and the performance.

When the actual time-delay is small, a bigger gain can be chosen to get a good performance. Otherwise, a middle gain can be chosen for a bigger time-delay.

\section{Acknowledgment}

This work was supported in part by Guangdong Key Laboratory of Clean Energy Technology in China (2008 A06031002). 


\section{References}

[1] E. Acha and M. Madrigal, Power Systems Harmonics, John Wiley \& Sons, Hoboken, NJ, USA, 2001.

[2] H. Fujita and H. Akagi, "A practical approach to harmonic compensation in power systems-series connection of passive and active filters," IEEE Transactions on Industry Applications, vol. 27, no. 6, pp. 1020-1025, 1991.

[3] Y. Xu, X. Xiao, H. Liu, and H. Wang, "Parallel operation of hybrid active power filter with passive power filter or capacitors," in Proceedings of the IEEE/PES Transmission and Distribution Conference and Exhibition: Asia and Pacific, pp. 16, August 2005.

[4] G. Chen, Z. Lu, and Z. Qian, "General principle of active power filter and its application," Proceedings of the CSEE, vol. 20, no. 9, pp. 17-21, 2000 (Chinese).

[5] L. Zhou, J. W. Jian, and L. W. Zhou, "Three-phase four-wire active power filter with one-cycle control," Proceedings of the CSEE, vol. 23, no. 3, pp. 85-88, 2003 (Chinese).

[6] J. K. Hale and S. M. Verduyn Lunel, Introduction to FunctionalDifferential Equations, vol. 99 of Applied Mathematical Sciences, Springer, New York, NY, USA, 1993.

[7] C. Tang, A. Luo, W. Zhao, and Z. K. Shuai, "Stability of hybrid shunt active power filter," Proceedings of the CSEE, vol. 28, no. 6, pp. 43-48, 2008 (Chinese).

[8] J. Hale, Theory of Functional Differential Equations, Springer, New York, NY, USA, 2nd edition, 1977.

[9] E. Fridman, "New Lyapunov-Krasovskii functionals for stability of linear retarded and neutral type systems," Systems \& Control Letters, vol. 43, no. 4, pp. 309-319, 2001.

[10] P. Park, "A delay-dependent stability criterion for systems with uncertain time-invariant delays," IEEE Transactions on Automatic Control, vol. 44, no. 4, pp. 876-877, 1999.

[11] M. da la Sen, "Quadratic stability and stabilization of switched dynamic systems with uncommensurate internal point delays," Applied Mathematics and Computation, vol. 185, no. 1, pp. 508526, 2007.

[12] M. Wu, Y. He, J.-H. She, and G.-P. Liu, "Delay-dependent criteria for robust stability of time-varying delay systems," Automatica, vol. 40, no. 8, pp. 1435-1439, 2004.

[13] Q.-L. Han, "Absolute stability of time-delay systems with sectorbounded nonlinearity," Automatica, vol. 41, no. 12, pp. 21712176, 2005.

[14] S. Boyd, L. El Ghaoui, E. Feron, and V. Balakrishnan, Linear Matrix Inequalities in System and Control Theory, vol. 15 of SIAM Studies in Applied Mathematics, Society for Industrial and Applied Mathematics (SIAM), Philadelphia, Pa, USA, 1994.

[15] K. Gu, V. L. Kharitonov, and J. Chen, Stability of Time-Delay Systems, Springer, New York, NY, USA, 2003.

[16] Q.-L. Han, "A discrete delay decomposition approach to stability of linear retarded and neutral systems," Automatica, vol. 45, no. 2, pp. 517-524, 2009. 


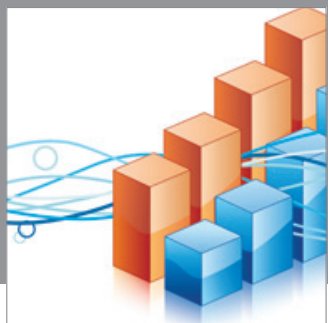

Advances in

Operations Research

mansans

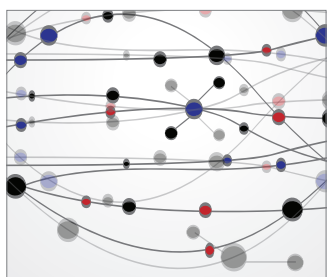

The Scientific World Journal
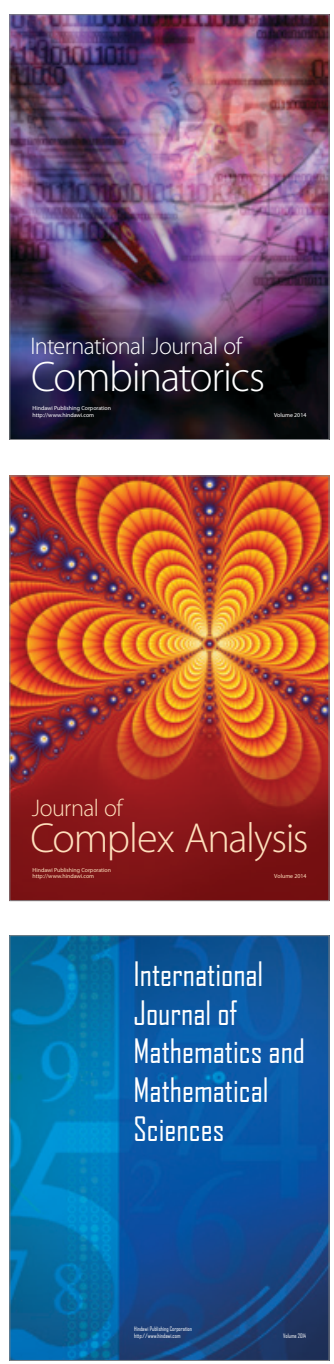
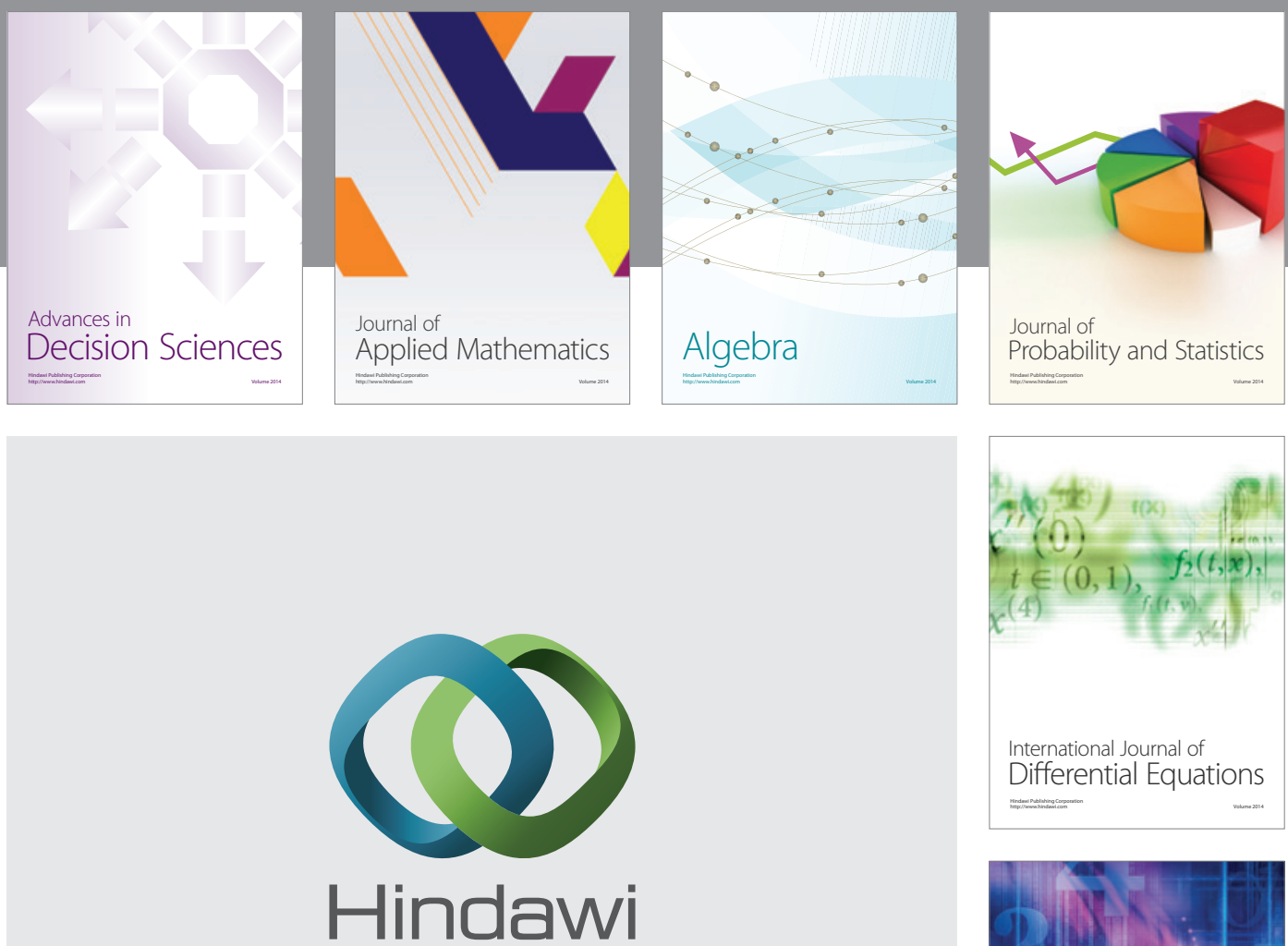

Submit your manuscripts at http://www.hindawi.com
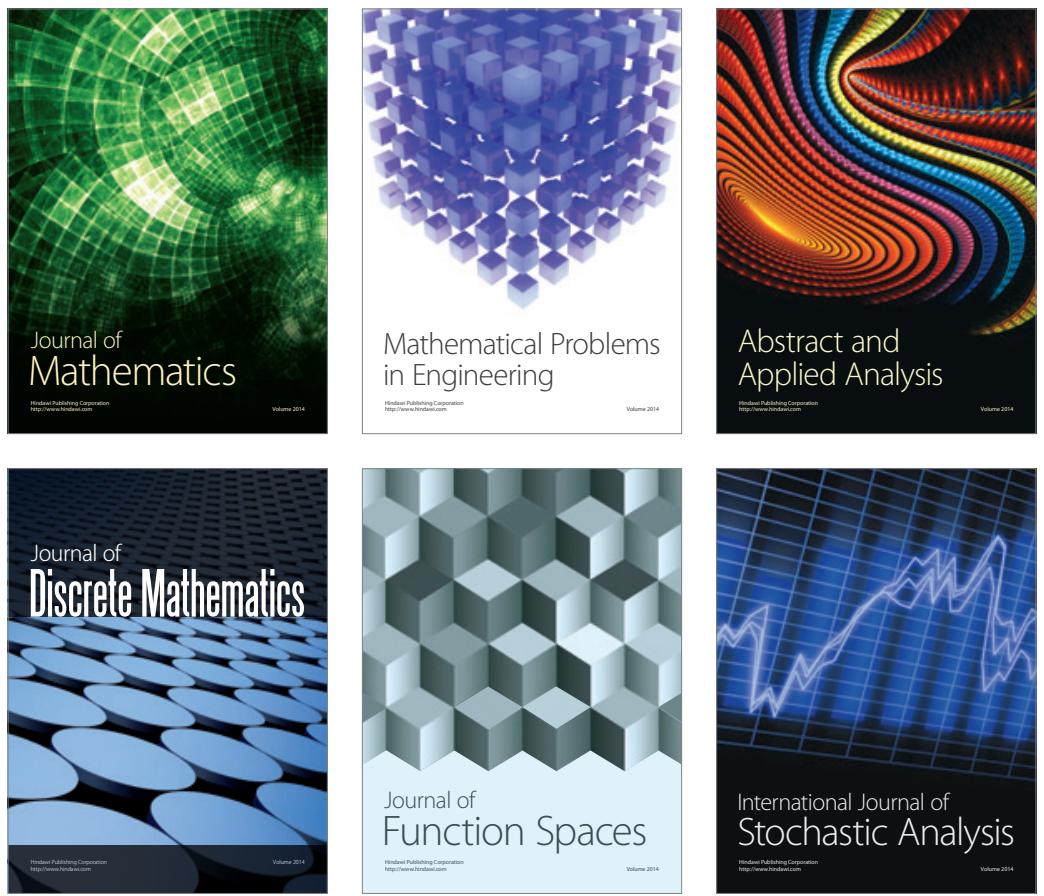

Journal of

Function Spaces

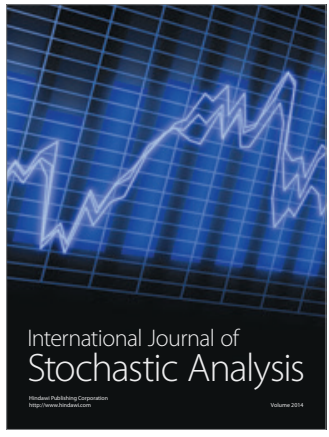

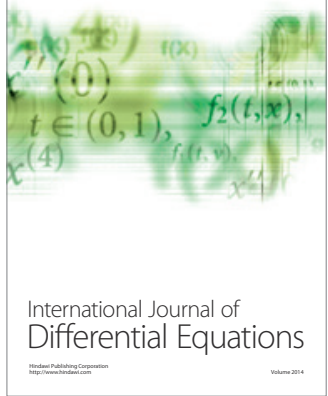
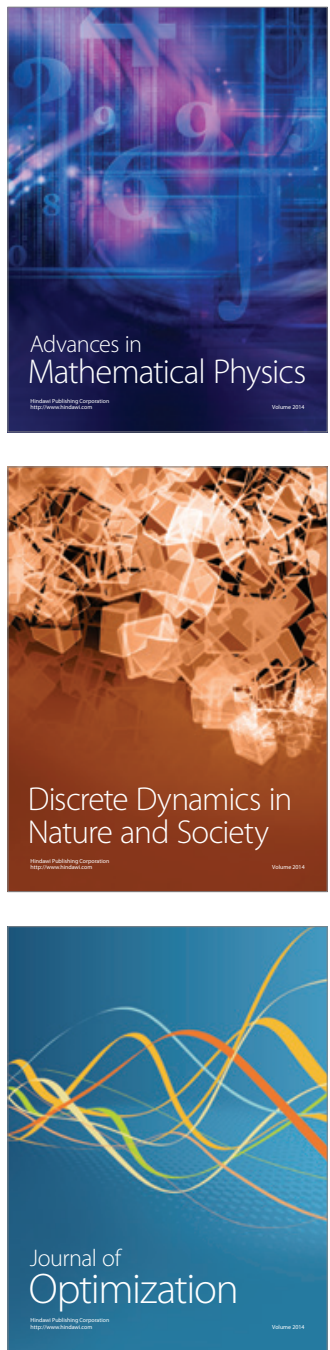